\title{
The Fast Wandering of Slow Birds
}

\author{
John Toner \\ Department of Physics and Institute of Theoretical Science, University of Oregon, Eugene, OR 97403, USA
}

(Dated: March 6, 2022)

\begin{abstract}
I study a single "slow" bird moving with a flock of birds of a different, and faster (or slower) species. I find that every "species" of flocker has a characteristic speed $\gamma \neq v_{0}$, where $v_{0}$ is the mean speed of the flock, such that, if the speed $v_{s}$ of the "slow" bird equals $\gamma$, it will randomly wander transverse to the mean direction of flock motion far faster than the other birds will: its mean-squared transverse displacement will grow in $d=2$ with time $t$ like $t^{\frac{5}{3}}$, in contrast to $t^{\frac{4}{3}}$ for the other birds. In $d=3$, the slow bird's mean squared transverse displacement grows like $t^{\frac{5}{4}}$, in contrast to $t$ for the other birds. If $v_{s} \neq \gamma$, the mean-squared displacement of the "slow" bird crosses over from $t^{\frac{5}{3}}$ to $t^{\frac{4}{3}}$ scaling in $d=2$, and from $t^{\frac{5}{4}}$ to $t$ scaling in $d=3$, at a time $t_{c}$ that scales according to $t_{c} \propto\left|v_{s}-\gamma\right|^{-2}$.

PACS numbers: 05.65.+b, 64.70.qj, 87.18.Gh
\end{abstract}

One of the most familiar and ubiquitous biological phenomena, flocking [1] - the collective, coherent motion of large numbers of organisms - also spans an enormous range of length scales: from kilometers (herds of wildebeest) to microns (e.g., the microorganism Dictyostelium discoideum [2-4]). Despite this familiarity, ubiquity, and enormous range of length scales, it is only in the last 15 years or so that the very rich and exotic scaling phenomena that occur in flocks has begun to be appreciated.

This new understanding began with Vicsek's [5] recognition that this phenomenon is a dynamical version of ferromagnetic ordering. Building on this insight, a phenomenological continuum theory of flocking [6 6$]$ was developed, which showed, among other things, that twodimensional flocks did not obey the Mermin-Wagner theorem [10]: that is, they are capable of spontaneously breaking a continuous symmetry (rotation invariance) by developing long-ranged order, even in flocks with only short ranged interactions. Such order is impossible (in spatial dimensions $d=2$ ) for equilibrium systems [10].

This fundamental difference between flocks and equilibrium systems arises from novel "anomalous" scalings of fluctuations in flocks. Many of the quantitative predictions of the phenomenological continuum theory about these scalings have been confirmed in numerical simulations [7, 11, 12].

One probe of this anomalous scaling that seems particularly apt to anyone who's ever watched a nature program in which wild animals are tagged with tracking devices (and who among us has not?) is to study the wandering of one particular creature flying along as part of a flock. The continuum theory [6 9] predicts that the lateral wandering of a bird in a flock of identical birds grows superdiffusively in $d=2$, scaling with time $t$ like $t^{\frac{2}{3}}$. The spectacular confirmation of this very striking prediction in numerical experiments [7, 12] remains one of the strongest tests to date of the analytic continuum theory.
However, such experiments only probe a small region of the full spatio-temporal range of flock motion; specifically, the regime of spatio-temporal points moving at the mean speed of the flock. It would clearly be a valuable, and more rigorous, test of the theory to probe other regions as well.

In this paper, I suggest one way to do so: by including a single "slow" bird, different from the other birds in the flock, which attempts to follow the other birds, but flies at a speed $v_{s} \neq v_{0}$, where $v_{0}$ is the average speed of the flock. I find that each species of bird has a certain "magic" speed $\gamma \neq v_{0}$ such that, if a "slow" bird of a different species flying at a speed $v_{s}=\gamma$ is added to the flock, it will wander laterally much faster even than the superdiffusive wandering of the others. This difference is a direct result of the different spatio-temporal regions probed by this bird because it is not moving at the average speed of the flock.

My result applies to any "ordered" flock moving on a substrate: i.e., one in which the flocking organisms spontaneously pick a direction to move together via purely short-ranged interactions that make neighbors tend to follow each other, but which do not pick out any a priori preferred direction for this motion. That is, the flocking spontaneously breaks rotation invariance, as equilibrium ferromagnetism does. Flocks moving without a substrate conserve momentum, and so have a very different hydrodynamics, which has been considered elsewhere 13. Whether or not slow birds wander rapidly in such a flock is an open question which remains uninvestigated, and which I will not address here.

One specific realization of such a system is one obeying the Vicsek algorithm [5], in which birds attempt to follow their near neighbors, but make mistakes. This algorithm has a parameter range in which the system "flocks" (i.e., has a non-zero average velocity: $\langle\vec{v}\rangle \neq \overrightarrow{0}$, where the average is over all the birds in a very large flock (i.e., in the thermodynamic limit)). In this "ordered" parameter 
range, the continuum theory [6 6 ] $]$ applies.

I will now briefly review the salient features of this continuum theory. This approach describes the flock by continuous, coarse grained number density $\rho(\vec{r}, t)$ and ve- locity $\vec{v}(\vec{r}, t)$ fields. The continuum equations of motion governing these fields can in the long-wavelength limit can be written down purely on symmetry grounds, and are: 6-9]

$$
\begin{aligned}
\partial_{t} \vec{v} & +\lambda_{1}(\rho,|\vec{v}|)(\vec{v} \cdot \vec{\nabla}) \vec{v}+\lambda_{2}(\rho,|\vec{v}|)(\vec{\nabla} \cdot \vec{v}) \vec{v}+\lambda_{3}(\rho,|\vec{v}|) \vec{\nabla}\left(|\vec{v}|^{2}\right)=\alpha(\rho,|\vec{v}|) \vec{v}-\beta(\rho,|\vec{v}|)|\vec{v}|^{2} \vec{v}-\vec{\nabla} P(\rho,|\vec{v}|) \\
& -\vec{v}\left(\vec{v} \cdot \vec{\nabla} P_{2}(\rho,|\vec{v}|)\right)+D_{B}(\rho,|\vec{v}|) \vec{\nabla}(\vec{\nabla} \cdot \vec{v})+D_{T}(\rho,|\vec{v}|) \nabla^{2} \vec{v}+D_{2}(\rho,|\vec{v}|)(\vec{v} \cdot \vec{\nabla})^{2} \vec{v}+\vec{f},
\end{aligned}
$$

$$
\frac{\partial \rho}{\partial t}+\nabla \cdot(\vec{v} \rho)=0
$$

where all of the parameters $\lambda_{i}(i=1 \rightarrow 3), \alpha, \beta$, $D_{B, T, 2}$ and the "isotropic Pressure" $P(\rho,|\vec{v}|)$ and the "anisotropic Pressure" $P_{2}(\rho,|\vec{v}|)$ are, in general, functions of the density $\rho$ and the magnitude $|\vec{v}|$ of the local velocity. It is useful to Taylor expand $P$ and $P_{2}$ around the equilibrium density $\rho_{0}$ :

$$
\begin{gathered}
P=P(\rho,|\vec{v}|)=\sum_{n=1}^{\infty} \sigma_{n}(|\vec{v}|)\left(\rho-\rho_{0}\right)^{n}, \\
P_{2}=P_{2}(\rho,|\vec{v}|)=\sum_{n=1}^{\infty} \mu_{n}(|\vec{v}|)\left(\rho-\rho_{0}\right)^{n} .
\end{gathered}
$$

Here $\beta, D_{B}, D_{2}$ and $D_{T}$ are all positive, and $\alpha<0$ in the disordered phase and $\alpha>0$ in the ordered state (in mean field theory).

The $\alpha$ and $\beta$ terms simply make the local $\vec{v}$ have a nonzero magnitude $v_{0}=\sqrt{\frac{\alpha}{\beta}}$ [14] in the ordered phase, where $\alpha>0$. $D_{B, T, 2}$ are the diffusion constants (or viscosities) reflecting the tendency of a localized fluctuation in the velocities to spread out because of the coupling between neighboring "birds". The $\vec{f}$ term is a random driving force representing the noise. It is assumed to be Gaussian with white noise correlations:

$$
<f_{i}(\vec{r}, t) f_{j}\left(\overrightarrow{r^{\prime}}, t^{\prime}\right)>=\Delta \delta_{i j} \delta^{d}\left(\vec{r}-\overrightarrow{r^{\prime}}\right) \delta\left(t-t^{\prime}\right),
$$

where $\Delta$ is a constant, and $i, j$ denote Cartesian components. The pressure $P$ tends, as in an equilibrium fluid, to maintain the local number density $\rho(\vec{r})$ at its mean value $\rho_{0}$, and $\delta \rho=\rho-\rho_{0}$. The "anisotropic pressure" $P_{2}(\rho,|\vec{v}|)$ in (11) is only allowed due to the non-equilibrium nature of the flock; in an equilibrium fluid such a term is forbidden, since Pascal's Law ensures that pressure is isotropic. In the nonequilibrium steady state of a flock, no such constraint applies. In earlier work [6 $[9]$ this term was ignored. While its inclusion does not affect the linearized theory of the ordered flock, it could, potentially, have important non-linear effects, as I discuss below.

The final equation (2) is just conservation of bird number: we don't allow our birds to reproduce or die on the wing. The interesting and novel results that arise when this constraint is relaxed by allowing birth and death while the flock is moving will be discussed elsewhere [15].

The hydrodynamic model embodied in equations (11), (3), and (2) is equally valid in both the "disordered" (i.e., non-moving) $(\alpha<0)$ and "ferromagnetically ordered" (i.e., moving) $(\alpha>0)$ state. Here I am interested in the "ferromagnetically ordered", broken-symmetry phase which occurs for $\alpha>0$. In this state, the velocity field can be written as:

$$
\vec{v}=v_{0} \hat{x}_{\|}+\overrightarrow{\delta v}=\left(v_{0}+\delta v_{\|}\right) \hat{x}_{\|}+\vec{v}_{\perp}
$$

where $v_{0} \hat{x}_{\|}=<\vec{v}>$ is the spontaneous average value of $\vec{v}$ in the ordered phase, and the fluctuations $\delta v_{\|}$and $\vec{v}_{\perp}$ of $\vec{v}$ about this mean velocity along and perpendicular to the direction of the mean velocity are assumed to be small. Indeed, I will be shortly be expanding the equation of motion (1) in these quantities. Taking $v_{0}=\sqrt{\frac{\alpha}{\beta}}$ as discussed above [14], and taking the dot product of both sides of equation (11) with $\vec{v}$ itself, I obtain:

$$
\begin{aligned}
\frac{1}{2}\left(\partial_{t}|\vec{v}|^{2}+\left(\lambda_{1}+2 \lambda_{3}\right)(\vec{v} \cdot \vec{\nabla})|\vec{v}|^{2}\right)+\lambda_{2}(\vec{\nabla} \cdot \vec{v})|\vec{v}|^{2} & =\left(\alpha-\beta|\vec{v}|^{2}\right)|\vec{v}|^{2}-\vec{v} \cdot \vec{\nabla} P-|\vec{v}|^{2} \vec{v} \cdot \vec{\nabla} P_{2}+D_{B} \vec{v} \cdot \vec{\nabla}(\vec{\nabla} \cdot \vec{v}) \\
& +D_{T} \vec{v} \cdot \nabla^{2} \vec{v}+D_{2} \vec{v} \cdot\left((\vec{v} \cdot \vec{\nabla})^{2} \vec{v}\right)+\vec{v} \cdot \vec{f}
\end{aligned}
$$


and time. (Indeed, the hydrodynamic equations (1) and (2) are only valid in this limit). Hence, terms involving space and time derivatives of $\overrightarrow{\delta v}(\vec{r}, t)$ and $\delta \rho(\vec{r}, t)$ are always negligible, in the hydrodynamic limit, compared to terms involving the same number of powers of fields without any time or space derivatives.

Furthermore, the fluctuations $\overrightarrow{\delta v}(\vec{r}, t)$ and $\delta \rho(\vec{r}, t)$ can themselves be shown to be small in the long-wavelength limit. Hence, we need only keep terms in (7) up to linear order in $\overrightarrow{\delta v}(\vec{r}, t)$ and $\delta \rho(\vec{r}, t)$. The $\vec{v} \cdot \vec{f}$ term can likewise be dropped, since it only leads to a term of order $\vec{v}_{\perp} f_{\|}$ in the $\vec{v}_{\perp}$ equation of motion, which is negligible (since $\vec{v}_{\perp}$ is small) relative to the $\overrightarrow{f_{\perp}}$ term already there.

These observations can be used to eliminate many of the terms in equation (7), and solve for the quantity

$$
U \equiv\left(\alpha(\rho,|\vec{v}|)-\beta(\rho,|\vec{v}|)|\vec{v}|^{2}\right)
$$

the solution is:

$$
U=\lambda_{2} \vec{\nabla} \cdot \vec{v}+\vec{v} \cdot \vec{\nabla} P_{2}+\frac{\sigma_{1}}{v_{0}} \partial_{\|} \delta \rho+\frac{1}{2 v_{0}}\left(\partial_{t}+\gamma_{2} \partial_{\|}\right) \delta v_{\|}
$$

Inserting this expression for $U$ back into equation (7) (where $U$ appears by virtue of its definition (8)), I find that $P_{2}$ and $\lambda_{2}$ cancel out of the $\vec{v}$ equation of motion, leaving

$$
\begin{aligned}
\partial_{t} \vec{v}+\lambda_{1}(\vec{v} \cdot \vec{\nabla}) \vec{v}+\lambda_{3} \vec{\nabla}\left(|\vec{v}|^{2}\right) & =\frac{\sigma_{1}}{v_{0}} \vec{v}\left(\partial_{\|} \delta \rho\right)-\vec{\nabla} P+D_{B} \vec{\nabla}(\vec{\nabla} \cdot \vec{v})+D_{T} \nabla^{2} \vec{v}+D_{2}(\vec{v} \cdot \vec{\nabla})^{2} \vec{v} \\
& +\left[\frac{1}{2 v_{0}}\left(\partial_{t}+\gamma_{2} \partial_{\|}\right) \delta v_{\|}\right] \vec{v}+\vec{f}
\end{aligned}
$$

This can be made into an equation of motion for $\vec{v}_{\perp}$ involving only $\vec{v}_{\perp}(\vec{r}, t)$ and $\delta \rho(\vec{r}, t)$ by projecting perpendicular to the direction of mean flock motion $\hat{x}_{\|}$, and eliminating $\delta v_{\|}$using equation(9) and the expansion

$$
U \approx-\Gamma_{1}\left(\delta v_{\|}+\frac{\left|\vec{v}_{\perp}\right|^{2}}{2 v_{0}}\right)-\Gamma_{2} \delta \rho
$$

where I've defined

$$
\Gamma_{1} \equiv-\left(\frac{\partial U}{\partial|\vec{v}|}\right)_{\rho}^{0}, \Gamma_{2} \equiv-\left(\frac{\partial U}{\partial \rho}\right)_{|\vec{v}|}^{0}
$$

with, here and hereafter, super- or sub-scripts 0 denoting functions of $\rho$ and $|\vec{v}|$ evaluated at $\rho=\rho_{0}$ and $|\vec{v}|=v_{0}$. I've also used the expansion (6) for the velocity in terms of the fluctuations $\delta v_{\|}$and $\vec{v}_{\perp}$ to write

$$
|\vec{v}|=v_{0}+\delta v_{\|}+\frac{\left|\vec{v}_{\perp}\right|^{2}}{2 v_{0}}+O\left(\delta v_{\|}^{2},\left|\vec{v}_{\perp}\right|^{4}\right)
$$

and kept only terms that an RG analysis shows to be relevant in the long wavelength limit. Inserting (11) into (9) gives:

$$
-\Gamma_{1}\left(\delta v_{\|}+\frac{\left|\vec{v}_{\perp}\right|^{2}}{2 v_{0}}\right)-\Gamma_{2} \delta \rho=\lambda_{2} \vec{\nabla}_{\perp} \cdot \vec{v}_{\perp}+\lambda_{2} \partial_{\|} \delta v_{\|}+\frac{\left(\mu_{1} v_{0}^{2}+\sigma_{1}\right)}{v_{0}} \partial_{\|} \delta \rho+\frac{1}{2 v_{0}}\left(\partial_{t}+\gamma_{2} \partial_{\|}\right) \delta v_{\|}
$$

where I've kept only linear terms on the right hand side of this equation, since the non-linear terms are at least of order derivatives of $\left|\vec{v}_{\perp}\right|^{2}$, and hence negligible, in the hydrodynamic limit, relative to the $\left|\vec{v}_{\perp}\right|^{2}$ term explicitly displayed on the left-hand side.
This equation can be solved iteratively for $\delta v_{\|}$in terms of $\vec{v}_{\perp}, \delta \rho$, and its derivatives. To lowest (zeroth) order in derivatives, $\delta v_{\|} \approx-\frac{\Gamma_{1}}{\Gamma_{2}} \delta \rho$. Inserting this into equation(14) everywhere it appears on the right hand side of that equation gives $\delta v_{\|}$to first order in derivatives: 


$$
\delta v_{\|} \approx-\frac{\Gamma_{1}}{\Gamma_{2}}\left(\delta \rho+\frac{1}{2 v_{0} \Gamma_{1}} \partial_{t} \delta \rho+\frac{\lambda_{4} \partial_{\|} \delta \rho}{\Gamma_{1}}\right)-\frac{\lambda_{2}}{\Gamma_{1}} \vec{\nabla}_{\perp} \cdot \vec{v}_{\perp}-\frac{\left|\vec{v}_{\perp}\right|^{2}}{2 v_{0}}
$$

Inserting (6), (13), and (15) into the equation of motion (10) for $\vec{v}$, and projecting that equation perpendic- ular to the mean direction of flock motion $\hat{x}_{\|}$and (2) for $\delta \rho$, gives, neglecting "irrelevant" terms:

$$
\begin{aligned}
\partial_{t} \vec{v}_{\perp}+\gamma \partial_{\|} \vec{v}_{\perp} & +\lambda_{1}^{0}\left(\vec{v}_{\perp} \cdot \vec{\nabla}_{\perp}\right) \vec{v}_{\perp}=-g_{1} \delta \rho \partial_{\|} \vec{v}_{\perp}-g_{2} \vec{v}_{\perp} \partial_{\|} \delta \rho-g_{3} \vec{v}_{\perp} \partial_{t} \delta \rho-\frac{c_{0}^{2}}{\rho_{0}} \vec{\nabla}_{\perp} \delta \rho-\sigma_{2} \vec{\nabla}_{\perp}\left(\delta \rho^{2}\right) \\
& +D_{B \text { eff }}^{0} \vec{\nabla}_{\perp}\left(\vec{\nabla}_{\perp} \cdot \vec{v}_{\perp}\right)+D_{T}^{0} \nabla_{\perp}^{2} \vec{v}_{\perp}+D_{\|}^{0} \partial_{\|}^{2} \vec{v}_{\perp}+\nu_{t} \partial_{t} \vec{\nabla}_{\perp} \delta \rho+\nu_{\|} \partial_{\|} \vec{\nabla}_{\perp} \delta \rho+\vec{f}_{\perp}
\end{aligned}
$$

where $D_{\rho} \equiv \rho_{0} \frac{\sigma_{1}}{\Gamma_{1}}, D_{B \text { eff }}^{0} \equiv D_{b}^{0}+\frac{2 v_{0} \lambda_{3} \lambda_{4}}{\Gamma_{1}}, D_{T}^{0}$, and $D_{\|}^{0} \equiv$ $D_{T}^{0}+D_{2}^{0} v_{0}^{2}$ are the diffusion constants, and I've defined

$$
\begin{gathered}
\gamma \equiv \lambda_{1}^{0} v_{0} \\
g_{1} \equiv\left(\frac{\partial \lambda_{1}}{\partial \rho}\right)_{0}-\frac{\Gamma_{2} \lambda_{1}}{\Gamma_{1}} \\
g_{2} \equiv \frac{\sigma_{1}}{v_{0}}-\frac{\left(\lambda_{1}+2 \lambda_{3}\right) \Gamma_{2}}{2 \Gamma_{1}} \\
g_{3} \equiv \frac{\Gamma_{2}}{2 v_{0} \Gamma_{1}}, \\
c_{0}^{2} \equiv \rho_{0} \sigma_{1}-\frac{2 \rho_{0} v_{0} \lambda_{3} \Gamma_{2}}{\Gamma_{1}} \\
D_{B \text { eff }} \equiv D_{B}^{0}+\frac{2 v_{0} \lambda_{2} \lambda_{3}}{\Gamma_{1}} \\
\nu_{t} \equiv \frac{\Gamma_{2} \lambda_{3}}{\Gamma_{1}^{2}}, \\
\nu_{2} v_{0} \lambda_{3} \lambda_{4}
\end{gathered}
$$

and, finally,

$$
D_{\|} \equiv D_{T}+D_{2} v_{0}^{2}
$$

It is extremely important to note that the speed $\gamma$ is not equal to the mean speed of the flock, since $\lambda_{1} \neq 1$. The reason that $\lambda_{1} \neq 1$ is the lack of Galilean invariance in the system under consideration, which in turn is due to the fact that we are considering flocks that move over a fixed surface, which provides a special rest frame.

This symmetry argument is confirmed by more microscopic, kinetic theory approaches 16 , which indeed find $\lambda \neq 1$. Furthermore, simulations can determine $\lambda_{1}$ by fitting the predictions of this hydrodynamic model to the observed correlation functions. The fits work extremely well, and find $\lambda_{1} \neq 1[7]$.

So $\gamma=\lambda_{1} v_{0} \neq v_{0}$; as we will see in a moment, this means that the identical birds making up a flock, which of course all move at speed $v_{0}$, will wander laterally (i.e., perpendicular to the direction of mean motion $\left.\hat{x}_{\|}\right)$much more slowly than a "slow" bird whose speed $v_{s}=\gamma$. Note that since no general argument requires $\lambda_{1}<1$, the "slow" bird might actually have to move faster than the ordinary birds, for some species.

Using (6) and (13) in the equation of motion (2) for $\rho$ gives, again neglecting irrelevant terms:

$$
\partial_{t} \delta \rho+\rho_{o} \vec{\nabla}_{\perp} \cdot \vec{v}_{\perp}+\vec{\nabla}_{\perp} \cdot\left(\vec{v}_{\perp} \delta \rho\right)+v_{2} \partial_{\|} \delta \rho=D_{\rho \|} \partial_{\|}^{2} \delta \rho+D_{\rho v} \partial_{\|}\left(\vec{\nabla}_{\perp} \cdot \vec{v}_{\perp}\right)+w_{1} \partial_{t} \partial_{\|} \delta \rho+w_{2} \partial_{\|}\left(\delta \rho^{2}\right)
$$

where I've defined:

$$
v_{2} \equiv v_{0}-\frac{\rho_{0} \Gamma_{2}}{\Gamma_{1}}
$$




$$
\begin{gathered}
w_{1} \equiv \frac{\Gamma_{2} \rho_{0}}{2 v_{0} \Gamma_{1}^{2}}, \\
D_{\rho \|} \equiv \frac{\Gamma_{2}}{\Gamma_{1}^{2}}\left(\frac{\lambda_{1}}{2}+\lambda_{2}+\lambda_{3}\right) \rho_{0}, \\
D_{\rho v} \equiv \frac{\lambda_{2} \rho_{o}}{\Gamma_{1}},
\end{gathered}
$$

and, last but not least,

$$
w_{2} \equiv \frac{\Gamma_{2}}{2 \Gamma_{1}}
$$

Linearizing (16) and (26), and spatio-temporally Fourier transforming them makes it straightforward algebra to calculate the hydrodynamic mode structure and spatio-temporally Fourier transformed correlation functions of the flock. (To obtain the latter simply involves using the assumed spatio-temporal noise correlations (5)).
The mode structure consists of a pair of propagating sound modes, with direction-dependent speeds given by: $c_{ \pm}\left(\theta_{\vec{q}}\right)$ are given by

$$
c_{ \pm}\left(\theta_{\vec{q}}\right)=\left(\frac{\gamma+v_{2}}{2}\right) \cos \left(\theta_{\vec{q}}\right) \pm c_{2}\left(\theta_{\vec{q}}\right)
$$

where I've defined

$$
c_{2}\left(\theta_{\vec{q}}\right) \equiv \sqrt{\frac{\left(\gamma-v_{2}\right)^{2} \cos ^{2}\left(\theta_{\vec{q}}\right)}{4}+c_{0}^{2} \sin ^{2}\left(\theta_{\vec{q}}\right)}
$$

where $\theta_{\vec{q}}$ is the angle between $\vec{q}$ and the direction of flock motion (i. e., the $x_{\|}$axis).

I'm particularly interested in the velocity autocorrelation, since, as I'll show in a moment, this determines the wandering of the birds relative to the mean motion of the flock. In the linearized approach just described, I find the spatio-temporally Fourier transformed velocity-velocity autocorrelation function is, ignoring negligible terms:

$$
\begin{aligned}
C_{\perp}(\vec{q}, \omega) \equiv\langle\vec{v}(\vec{q}, \omega) \cdot \vec{v}(-\vec{q},-\omega)\rangle & =\frac{\Delta\left(\omega-v_{2} q_{\|}\right)^{2}}{\left[\left(\omega-c_{+}\left(\theta_{\vec{q}}\right) q\right)^{2}+\epsilon_{+}^{2}(\vec{q})\right]\left[\left(\omega-c_{-}\left(\theta_{\vec{q}}\right) q\right)^{2}+\epsilon_{-}^{2}(\vec{q})\right]} \\
& +\frac{(d-2) \Delta}{\left[\left(\omega-\gamma q_{\|}\right)^{2}+\Gamma_{T}^{2}(\vec{q})\right]}
\end{aligned}
$$

where I've defined

with

$$
\epsilon_{ \pm} \equiv \frac{\mathrm{NUM}}{\left(2 \mathrm{c}_{ \pm}\left(\theta_{\overrightarrow{\mathrm{q}}}\right)-\left(\mathrm{v}_{2}+\gamma\right) \cos \left(\theta_{\overrightarrow{\mathrm{q}}}\right)\right)}
$$

$$
\begin{aligned}
\mathrm{NUM} & \equiv\left(\Gamma_{L}(\vec{q})+\Gamma_{\rho}(\vec{q})-w_{1} c_{ \pm}\left(\theta_{\vec{q}}\right) \cos \left(\theta_{\vec{q}}\right) q^{2}\right) c_{ \pm}\left(\theta_{\vec{q}}\right)-v_{2} \Gamma_{L}(\vec{q}) \cos \left(\theta_{\vec{q}}\right)-\gamma\left(\Gamma_{\rho}(\vec{q})-w_{1} c_{ \pm}\left(\theta_{\vec{q}}\right) \cos \left(\theta_{\vec{q}}\right) q^{2}\right) \cos \left(\theta_{\vec{q}}\right) \\
& +\frac{c_{0}^{2}}{\rho_{0}} D_{\rho v} \frac{q_{\|} q_{\perp}^{2}}{q}-\rho_{0} q_{\perp}^{2}\left(\nu_{t} c_{ \pm}\left(\theta_{\vec{q}}\right)+\nu_{\|} \cos \left(\theta_{\vec{q}}\right)\right)
\end{aligned}
$$

and the wavevector dependent longitudinal, transverse, and $\rho$ dampings $\Gamma_{L, \rho}$ are given by:

$$
\begin{aligned}
& \Gamma_{L}(\vec{q})=D_{L} q_{\perp}^{2}+D_{\|}^{0} q_{\|}^{2}, \\
& \Gamma_{T}(\vec{q})=D_{T}^{0} q_{\perp}^{2}+D_{\|}^{0} q_{\|}^{2},
\end{aligned}
$$

and

$$
\Gamma_{\rho}(\vec{q})=D_{\rho \|} q_{\|}^{2}
$$

with $D_{L} \equiv D_{B \text { eff }}^{0}+D_{T}^{0}$.

The first term in equation 35 comes from the "longitudinal" component $v_{L}$ of $\vec{v}_{\perp}$, by which I mean the component along $\vec{q}_{\perp}$, while the second comes from the $d-2$ "transverse" components of $\vec{v}_{\perp}$ which are perpendicular to $\vec{q}_{\perp}$. Clearly, in $d=2$, only the longitudinal component is present; this is why the second (transverse) term in (34) vanishes in $d=2$.

What about the effect of the non-linear terms in (16) and (26)? In spatial dimensions $d<4$, these terms are 
strongly relevant in the RG sense [] - [9]

What "relevant in the RG sense" means in plain English is that these non-linear terms lead to different scaling behavior at long distances and times than predicted by the linearized version of (16) and (26), which of course ignores those terms.

Determining quantitatively this modified scaling is difficult; indeed, the only known analytic approach is an $\epsilon \equiv 4-d$ expansion about the critical dimension $d=4$, which clearly tells us little about what goes on in $d=2$.

However, there are many examples known in which non-linearities that become relevant for $d$ just below 4 become irrelevant again as $d$ is decreased to 3 , and remain so down to $d=2$ (e.g., cubic symmetry breaking terms at the phase transition of the $X Y(\mathrm{O}(2))$ model[17]).

Now, in our problem, it is impossible for all of the non-linearities in (16) and (26) to be irrelevant in any $d<4$, since the linearized fixed point can be shown to be unstable against all of these nonlinearities for those $d$ 's. However, it is quite possible, as illustrated by the discussion of the previous paragraph, that the ordered phase of the flock is controlled by a fixed point in which only one of these non-linearities is important. While there is no analytic calculation so far that shows this is the case, if we conjecture that the dominant non-linearity in equation (16) is $\lambda_{1}^{0}$, and that all of the other non-linearities are irrelevant, in the RG sense, below $d=4$ (a result which is, at least, not ruled out by the $4-\epsilon$ calculation that has been done), then one can show [6]-[9] that the effect of these non-linearities on all spatio-temporally Fourier transformed two point correlation functions can be incorporated entirely by replacing $D_{T}^{0}$ and $D_{B \text { eff }}$ everywhere they appear in the linearized expressions for those correlation functions with renormalized, strongly wavevector $(\vec{q})$-dependent diffusion coefficients $D_{T, B}(\vec{q})$ that diverge as $\vec{q} \rightarrow \overrightarrow{0}$ according to the scaling law:

$$
\begin{gathered}
D_{T, B}(\vec{q})=q_{\perp}^{2 \zeta-2} f_{T, B}\left(\frac{\left(\frac{q_{\|}}{\Lambda}\right)}{\left(\frac{q_{\perp}}{\Lambda}\right)^{\zeta}}\right) \\
\propto \begin{cases}\mathrm{q}_{\perp}^{2 \zeta-2}, & \frac{q_{\|}}{\Lambda} \ll\left(\frac{q_{\perp}}{\Lambda}\right)^{\zeta} \\
q_{\|}-\frac{2}{\zeta}, & \frac{q_{\|}}{\Lambda} \gg\left(\frac{q_{\perp}}{\Lambda}\right)^{\zeta}\end{cases}
\end{gathered}
$$

here $\Lambda$ is an ultraviolet cutoff of order an inverse interbird distance, and the universal exponent $\zeta$ is given by:

$$
\zeta=\frac{d+1}{5}
$$

In addition, $\Gamma_{\rho}(\vec{q})$ is also modified, becoming

$$
\Gamma_{\rho}(\vec{q})=D_{\rho \|} q_{\|}^{2}+D_{\rho \perp}(\vec{q}) q_{\perp}^{2},
$$

where the new diffusion coefficient $D_{\rho \perp}(\vec{q})$ also becomes anomalous (i.e., a divergent function of wavevector $\vec{q}$ as $\vec{q} \rightarrow \overrightarrow{0}$; indeed, it obeys the same scaling law as $D_{T, B}$; i.e.,

$$
\begin{aligned}
& D_{\rho \perp}(\vec{q})=q_{\perp}^{2 \zeta-2} f_{\rho \perp}\left(\frac{\left(\frac{q_{\|}}{\Lambda}\right)}{\left(\frac{q}{\Lambda}\right)^{\zeta}}\right) \\
& \propto \begin{cases}q_{\perp}^{2 \zeta-2}, & \frac{q_{\|}}{\Lambda} \ll\left(\frac{q_{\perp}}{\Lambda}\right)^{\zeta} \\
q_{\|}^{2-\frac{2}{\zeta}}, & \frac{q_{\|}}{\Lambda} \gg\left(\frac{q_{\perp}}{\Lambda}\right)^{\zeta}\end{cases}
\end{aligned}
$$

As a result of the divergences of $D_{B, T, \rho \perp}(\vec{q})$, the dampings $\Gamma_{L, T, \rho}(\vec{q})$ also obey scaling laws:

$$
\begin{gathered}
\Gamma_{L, T, \rho}(\vec{q})=q_{\perp}^{2 \zeta} f_{\Gamma_{L}, \Gamma_{T}, \Gamma_{\rho}}\left(\frac{\left(\frac{q_{\|}}{\Lambda}\right)}{\left(\frac{q_{\perp}}{\Lambda}\right)^{\zeta}}\right) \\
\propto \begin{cases}q_{\perp}^{2 \zeta}, & \frac{q_{\|}}{\Lambda} \ll\left(\frac{q_{\perp}}{\Lambda}\right)^{\zeta} \\
q_{\|}, & \frac{q_{\|}}{\Lambda} \gg\left(\frac{q_{\perp}}{\Lambda}\right)^{\zeta} .\end{cases}
\end{gathered}
$$

While the reader may with some justice be skeptical that we would be so lucky as to have the only relevant non-linearity in the problem prove to be the only one that we can handle analytically, there is considerable evidence from simulations [7, 8, 11, 12] supporting the "canonical" behavior just described, in both $d=2$ and $d=3$. I will therefore assume for the remainder of this paper that this "canonical" behavior is the true behavior of a flock.

The divergence as $\vec{q} \rightarrow \overrightarrow{0}$ of the diffusion coefficients $D_{B, T, \rho \perp}(\vec{q})$ embodied in equations (40) and (43) has dramatic consequences for the velocity fluctuations. In particular, it implies that, for fixed $|\vec{q}|$, these fluctuations will damp out far more slowly for $\vec{q}$ along the direction of mean flock motion $\hat{x}_{\|}$than for any other direction of $\vec{q}$. More precisely, those modes with $\frac{q_{\|}}{\Lambda} \sim\left(\frac{q_{\perp}}{\Lambda}\right)^{\zeta}$ will decay far more slowly than those with $\frac{q_{\|}}{\Lambda} \ll\left(\frac{q_{\perp}}{\Lambda}\right)^{\zeta}$. This means that, as $\vec{q} \rightarrow \overrightarrow{0}$, the largest fluctuations will occur at wavevectors $\vec{q}$ with $q_{\|} \gg q_{\perp}$, since $\zeta<1$. Thus, we only need to evaluate $C_{\perp}(\vec{q}, \omega)$ for those values of $\vec{q}$ with $q_{\|} \gg q_{\perp}$. In this limit, the autocorrelation function (34) can be considerably simplified, to:

$$
C_{\perp}(\vec{q}, \omega) \approx \frac{\Delta}{\left[\left(\omega-\gamma q_{\|}-\frac{c_{0}^{2} q_{\perp}^{2}}{\left(\gamma-v_{2}\right) q_{\|}}\right)^{2}+\Gamma_{L}^{2}(\vec{q})\right]}+\frac{(d-2) \Delta}{\left[\left(\omega-\gamma q_{\|}\right)^{2}+\Gamma_{T}^{2}(\vec{q})\right]}
$$

Further simplification can be made by comparing the

$c_{0}^{2} q_{\perp}^{2}$ term in the first denominator with the $\Gamma_{L}^{2}(\vec{q})$ term 
therein. The ratio of these scales like

$$
\frac{q_{\perp}^{4}}{q_{\|}^{2} \Gamma_{L}^{2}(\vec{q})} \propto \frac{q_{\perp}^{2(1-\zeta)}}{q_{\|}^{2} f_{\Gamma_{L}}\left(\frac{\left(\frac{q_{\|}}{\Lambda}\right)}{\left(\frac{q_{\perp}}{\Lambda}\right)^{\zeta}}\right)}
$$

For the important regime of wavevectors $q_{\perp}^{\zeta} \sim q_{\|}$, this ratio scales 20] like $q_{\perp}^{2-3 \zeta}$, and, hence, vanishes at small $q_{\perp}$ if and only if the exponent $\zeta<\frac{2}{3}$. From equation (41), it's clear that this condition on $\zeta$ is satisfied in $d=2$, but not $d=3$. Thus, in $d=2$, where the second term in (34) vanishes, we can drop the $c_{0}^{2} q_{\perp}^{2}$ term in (45), and obtain the much simpler expression:

$$
C_{\perp}(\vec{q}, \omega) \approx \frac{\Delta}{\left[\left(\omega-\gamma q_{\|}\right)^{2}+\Gamma_{L}^{2}(\vec{q})\right]} \quad(d=2) .
$$

In $d=3$, on the other hand, the $c_{0}^{2} q_{\perp}^{2}$ term in (45) dominates the $\Gamma_{L}(\vec{q})$ term, since $\zeta=\frac{4}{5}$ in $d=3$. In this case, the first (i.e., the longitudinal) term in (45) is, throughout most of the important range of $\vec{q}$, much smaller than it would be in the absence of this term. On the other hand, the second, $d-2$ term in (45) is comparable in magnitude to what the first term would have been, had I dropped the $c_{0}^{2} q_{\perp}^{2}$ term. To see this, simply note that the two terms would then become identical, except for the factor of $d-2$, which is 1 in $d=3$, and the replacement of $\Gamma_{L}$ by $\Gamma_{T}$. Since $\Gamma_{L}$ and $\Gamma_{T}$ both scale the same way with $\vec{q}$, this latter change makes little difference. Hence, keeping the $c_{0}^{2} q_{\perp}^{2}$ term makes the first (longitudinal) term in (45) actually much smaller than the second, transverse, one. Hence we can drop that first term, and write:

$$
C_{\perp}(\vec{q}, \omega) \approx \frac{\Delta}{\left[\left(\omega-\gamma q_{\|}\right)^{2}+\Gamma_{T}^{2}(\vec{q})\right]} \quad(d=3) .
$$

Now let us add to this ordered flock a single "slow" bird [18] which tries to follow the other birds in the flock via some short-ranged interaction, but which moves at a different speed $v_{s}$ than the other birds in the flock. For example, one could imagine (or simulate) a Vicsek algorithm [5] in which the "slow" bird obeys the same algorithm as the others, except that its speed of motion between time steps differs from those of the other birds.

Consider now the mean squared lateral displacement of the slow bird:

$$
w^{2}\left(t ; v_{s}\right) \equiv\left\langle\left|\vec{x}_{s}^{\perp}(t)-\vec{x}_{s}^{\perp}(0)\right|^{2}\right\rangle
$$

perpendicular to the mean direction of motion of the flock. (Here and throughout this paper, $\perp$ and $\|$ denote directions perpendicular to, and along, the direction of mean flock motion, respectively.)

Since the mean $\vec{x}_{\perp}-$ position $\vec{x}_{s}^{\perp}$ of the slow bird obeys

$$
\vec{x}_{s}^{\perp}(t)=\vec{x}_{s}^{\perp}(0)+\int_{0}^{t} \vec{v}_{s}^{\perp}(t) d t
$$

where $v_{s}^{\perp}(t)$ is the $\perp$ velocity of the slow bird at time $t$, $w^{2}\left(t ; v_{s}\right)$ is given by

$$
w^{2}\left(t ; v_{s}\right)=\int_{0}^{t} d t^{\prime} \int_{0}^{t} d t^{\prime \prime}\left\langle\vec{v}_{s}^{\perp}\left(t^{\prime}\right) \cdot \vec{v}_{s}^{\perp}\left(t^{\prime \prime}\right)\right\rangle .
$$

Now I need to relate the perpendicular velocity of the slow bird to the position and time dependent velocity field $\vec{v}_{\perp}(\vec{r}, t)$ of the flock. Since the slow bird moves in the same direction [21] as the other birds in the flock, the required perpendicular velocity is given by:

$$
\vec{v}_{s}^{\perp}(t)=\frac{\vec{v}_{\perp}\left(\vec{r}_{s}(t), t\right) v_{s}}{v_{0}}
$$

where $v_{0}$ is the mean speed of the flock (in the sense that, $<\vec{v}(\vec{r}, t)>=v_{0} \hat{x}_{\|}$, with $\hat{x}_{\|}$the unit vector along the direction of mean flock motion), and $\vec{r}_{s}(t)$ is the position of the slow bird at time $t$. This is given by

$$
\vec{r}_{s}(t)=\vec{r}_{s}(0)+v_{s} t \hat{x}_{\|}+\delta x_{s}^{\|}(t) \hat{x}_{\|}+\overrightarrow{\delta x}_{s}^{\perp}(t)
$$

where $\delta x_{s}^{\|}(t)$ and $\overrightarrow{\delta x_{s}^{\perp}}(t)$ are the deviations of the slow bird from uniform motion at speed $v_{s}$ along $\hat{x}_{\|}$.

Using (52) and (53), I see that the desired single bird autocorrelation function in (51) is:

$$
\begin{aligned}
\left\langle\vec{v}_{s}^{\perp}\left(t^{\prime}\right) \cdot \vec{v}_{s}^{\perp}\left(t^{\prime \prime}\right)\right\rangle & \left.=\left(\frac{v_{s}}{v_{0}}\right)^{2}\left\langle\vec{v}_{\perp}\left(\vec{r}_{s}\left(t^{\prime}\right), t\right) \cdot \vec{v}_{\perp}\left(\vec{r}_{s}\left(t^{\prime \prime}\right), t^{\prime \prime}\right)\right)\right\rangle \\
& =\left(\frac{v_{s}}{v_{0}}\right)^{2} C_{\perp}\left(\overrightarrow{\delta x}_{s}^{\perp}\left(t^{\prime}\right)-\overrightarrow{\delta x}_{s}^{\perp}\left(t^{\prime \prime}\right), v_{s}\left(t^{\prime}-t^{\prime \prime}\right)+\delta x_{s}^{\|}\left(t^{\prime}\right)-\delta x_{s}^{\|}\left(t^{\prime \prime}\right), t^{\prime}-t^{\prime \prime}\right)
\end{aligned}
$$

where $C_{\perp}\left(\vec{r}_{\perp}, r_{\|}, t\right)$ is the real space and time perpendicular velocity field auto-correlation function, and I've used

$$
\vec{r}_{s}(t)=\vec{r}_{s}(0)+\left(v_{s} t+\delta x_{s}^{\|}(t)\right) \hat{x}_{\|}+\overrightarrow{\delta x}_{s}^{\perp}(t),
$$


to write both $\vec{r}_{s}\left(t^{\prime}\right)$ and $\vec{r}_{s}\left(t^{\prime \prime}\right)$.

I'll assume (and have verified a posteriori) that both $\delta x_{s}^{\|}$and $\delta x_{s}^{\perp}$ are small enough compared to the average motion $v_{s} t \hat{x}_{\|}$that their effect on the velocityvelocity autocorrelation in (54) is negligible. This will be true if $w^{2}(t) \ll t^{2}$, which, as I will show a posteriori, it is. This leaves the task of evaluating $C(\delta t) \equiv$ $C_{\perp}\left(R_{\perp}=0, R_{\|}=v_{s} \delta t, \delta t\right)$, where I've defined $\delta t \equiv t^{\prime}-$ $t^{\prime \prime}$. Expressing $C_{\perp}$ in terms of its spatio-temporal Fourier transform equation (34) gives

$$
C(\delta t) \equiv\left\langle\vec{v}_{s}^{\perp}\left(t^{\prime}\right) \cdot \vec{v}_{s}^{\perp}\left(t^{\prime \prime}\right)\right\rangle=\left(\frac{v_{s}}{v_{0}}\right)^{2} C_{\perp}\left(R_{\perp}=0, R_{\|}=v_{s} \delta t\right)=\left(\frac{v_{s}}{v_{0}}\right)^{2} \int \frac{d^{d-1} q_{\perp} d q_{\|} d \omega}{(2 \pi)^{d+1}} e^{i\left(\omega-v_{s} q_{\|} \delta t\right)} C_{\perp}(\vec{q}, \omega)
$$

Performing the integral over $\omega$ by complex contour techniques, and using equation (47) for $C_{\perp}(\vec{q}, \omega)$ in $d=2$, and (48) for $C_{\perp}(\vec{q}, \omega)$ in $d=3$, gives

$$
C(\delta t)=\left(\frac{v_{s}}{v_{0}}\right)^{2} \Delta \int \frac{d^{d} q}{(2 \pi)^{d}} \frac{\exp \left\{i\left(\left(v_{s}-\gamma\right) q_{\|}\right) \delta t-\Gamma_{\sigma}(\vec{q}) \delta t\right\}}{\Gamma_{\sigma}(\vec{q})}
$$

where $\sigma=L$ in $d=2$, and $\sigma=T$ in $d=3$.

Now clearly, if $v_{s} \neq \gamma$, the first term in the exponential is much larger, at small $q$, than the $\Gamma_{L}(\vec{q})$ term, since the former scales as $q_{\|}$, while the latter scales as $q_{\|}^{2}$, in the dominant regime $q_{\|} \sim q_{\perp}^{\zeta}$. Hence, I can drop the latter term in the exponential, since it is small $q$ 's which dominate the integral at large times (as we'll see in a moment). Dropping this term, and using the scaling law (44) for $\Gamma_{\sigma}(\vec{q})$, it is easy to see that rescaling the components $q_{\|}$and $\vec{q}_{\perp}$ of $\vec{q}$ according to

$$
q_{\|} \equiv \frac{Q_{\|}}{\delta t}, \quad \vec{q}_{\perp} \equiv \frac{\vec{Q} \perp}{\delta t^{\frac{1}{\zeta}}}
$$

pulls all of the time dependence out of the integral $d^{d} q$, giving the scaling law

$$
C(\delta t) \propto \delta t^{\frac{1-d}{\zeta}-1+2} \propto \delta t^{\phi(d)}
$$

for $C(\delta t)$, where I've defined

$$
\phi(d) \equiv \frac{1-d}{\zeta}+1
$$

It is important to note that this scaling law for $C(\delta t)$ only holds for $\delta t$ large, since it is only for such times that the hydrodynamic theory is valid. Thus, we are not concerned with any apparent divergences at short times that occur when (59) is inserted into (51). Divergences as $t \rightarrow \infty$ are real, on the other hand; these will occur whenever $\phi(d)$, as given by equation (60), is $>-1$.

This happens in $d=2$, where equation (60) gives $\phi(2)=-\frac{2}{3}$; inserting this into (51) gives

$$
w^{2}\left(t ; v_{s}\right) \propto t^{\frac{4}{3}} \quad(\text { ordinary birds, } \mathrm{d}=2),
$$

a known 7] result for birds in flocks of identical birds in $d=2$. In $d=3$, on the other hand, equation (60) gives $\phi(2)=-\frac{3}{2}<-1$. In this case, the integral over $t^{\prime \prime}$ in (51) converges at large $\left|t^{\prime}-t^{\prime \prime}\right|$, and so is dominated by short times, where, as just discussed, the hydrodynamic theory is invalid. Thus, the result of performing the integral over $t^{\prime \prime}$ in (51) is a constant, independent of $t$ and $t^{\prime}$. As a result, the integral over $t^{\prime}$ in (51) is just

$$
w^{2}\left(t ; v_{s}\right) \propto t \quad(\text { ordinary birds, } \mathrm{d}=3),
$$

another already known result [7]. Note that these results apply not only to the identical birds that make up the bulk of the flock, but to any slow bird added to the flock whose speed $v_{s} \neq \gamma$.

What happens if $v_{s}=\gamma$ ? Now things change dramatically, since the $\left(v_{s}-\gamma\right) q_{\|} t$ term in the exponential in equation (57) vanishes. As a result, I must keep the $\Gamma_{\sigma}$ term in the exponential in that equation. Doing so, and again using equation (44), I see that the rescaling to a new vector variable $\vec{Q}$ via

$$
\begin{gathered}
q_{\|} \equiv \frac{Q_{\|}}{\sqrt{\delta t}} \\
\vec{q}_{\perp} \equiv \frac{\vec{Q}_{\perp}}{\delta t^{\frac{1}{2 \zeta}}}
\end{gathered}
$$

pulls all of the time dependence out of the integral when $v_{s}=\gamma$, and gives the scaling law

$$
C(\delta t)=A \delta t^{\frac{\phi(d)}{2}},
$$


where $A$ is an unimportant constant.

Note that this decay is much slower than that given in equation(59) for a bird moving at a speed other than the magic speed $v_{s}=\gamma$. Note further that since, in general, the mean speed $v_{0}$ of the flock differs from $\gamma$ (i.e., $v_{0} \neq \gamma$ ) the identical birds that make up the bulk of the flock will exhibit the more rapidly decaying correlations of equation(59). It is only a bird that differs from the generic members of the flock, specifically by flying at a speed $v_{s} \neq v_{0}$, that has a chance to achieve $v_{s}=\gamma$, and the much slower decay equation (65).

This slower decay of velocity correlations leads immediately to faster lateral wandering for birds moving at this magic speed $\gamma$, in both $d=2$ and $d=3$, since the exponent $\frac{\phi(d)}{2}$ is $>-1$ in both dimensions. As a result, the integral over $t^{\prime}$ and $t^{\prime \prime}$ is dominated by large times, and I find, by inserting (65) into (51),

$$
\begin{gathered}
w^{2}\left(t ; v_{s}=\gamma\right)=A \int_{0}^{t} d t^{\prime} \int_{0}^{t} d t^{\prime \prime}\left|t^{\prime}-t^{\prime \prime}\right|^{\frac{\phi(d)}{2}} \\
\propto t^{\frac{5}{2}-\left(\frac{1-d}{2 \zeta}\right)}=t^{\frac{1}{\zeta}} \quad(\text { slow bird, general } \mathrm{d}<4),
\end{gathered}
$$

where in the last equality I've used equation (41) for $\zeta$.

Using equation (41) again, I get, in $d=2$ spatial dimensions,

$$
w^{2}\left(t ; v_{s}\right) \propto \begin{cases}t^{\frac{5}{3}}, & v_{s}=\gamma, \\ t^{\frac{4}{3}}, & v_{s} \neq \gamma \quad(d=2),\end{cases}
$$

while in $d=3$,

$$
w^{2}\left(t ; v_{s}\right) \propto \begin{cases}t^{\frac{5}{4}}, & v_{s}=\gamma \\ t & v_{s} \neq \gamma \quad(d=3),\end{cases}
$$

In both dimensions, birds moving at the magic speed $\gamma$ wander laterally much faster than birds moving at any other speed. In particular, they wander much faster than the "normal" birds in the flock.

What happens to a bird moving at a speed $v_{s}$ close to, but not equal to, $\gamma$ ? For sufficiently short times, it should be impossible to tell that this bird was not moving at exactly the magic speed $\gamma$, and would therefore expect to recover the $w^{2} \propto t^{\frac{1}{\zeta}}$ scaling I just found for that case. For longer times, though, one would expect the difference between the speed $v_{s}$ of the slow bird and the magic speed $\gamma$ to become apparent, leading to a crossover to the $w^{2} \propto t^{\frac{4}{3}}$ scaling found in that case.

This proves to be precisely the case. To see this, note that the rescaling equations (63), (64) imply that the integral in equation (57) for $C\left(t ; v_{s}\right)$ is dominated by parallel wavevectors

$$
q_{\|}^{d o m} \propto t^{-\frac{1}{2}}
$$

Comparing the $\delta v q_{\|}$term in the exponent of equation (57) with the $\Gamma_{L}$ term there, where $\delta v \equiv v_{s}-\gamma$, and using the scaling law (43) for $\Gamma_{L}$ and (69) for the dominant $q$,
I see that $\delta v q_{\|}^{d o m} \sim \Gamma_{L}\left(\vec{q}_{d o m}\right)$ at a time $t_{c}$ which scales according to $\delta v t_{c}^{-\frac{1}{2}} \propto\left(t_{c}^{-\frac{1}{2}}\right)^{2}$, which implies

$$
t_{c}(\delta v) \propto \delta v^{-2} .
$$

For smaller times $\left(t \ll t_{c}\right)$, the $\Gamma_{L}$ term in equation (??) dominates the $\delta v q_{\|}$term, and the $w^{2}(t) \propto t^{\frac{1}{\zeta}}$ law found in eqn (66) holds. In the opposite limit $\left(t \gg t_{c}\right)$, the $v_{s} \neq \gamma$ scaling holds.

To summarize, for flocks in $d=2$ spatial dimensions,

$$
w^{2}\left(t ; v_{s}\right) \propto\left\{\begin{array}{l}
t^{\frac{5}{3}}, t \ll t_{c}(\delta v) \\
t^{\frac{4}{3}}, t \gg t_{c}(\delta v)
\end{array}\right.
$$

while for $d=3$,

$$
w^{2}\left(t ; v_{s}\right) \propto \begin{cases}t^{\frac{5}{4}}, & t \ll t_{c}(\delta v) \\ t, & t \gg t_{c}(\delta v)\end{cases}
$$

with $t_{c}(\delta v)$ given by (70).

Numerical experiments could test this result by doing a series of simulations with added slow birds moving at different speeds. The magic speed $\gamma$ can be determined by fitting the above expressions. Alternatively, one can independently determine $\gamma$ by measuring the directiondependent sound mode propagation speed in a simulation, and fitting the result to equation (32), as was done in [7]. An easier alternative for simulators who, understandably, do not wish to undertake this considerable numerical task, would be to simply simulate exactly the same model as was used in 7], with the addition of a single slow bird; in this case, one could simply use the value of $\gamma$ determined by [7].

In the (potentially simulate-able) case of spatial dimensions $d \geq 4$, I find simple "drunkard's walk" wandering $\left(w^{2}\left(t ; v_{s}\right) \propto t\right)$ independent of $v_{s}$.

I thank Sriram Ramaswamy, Yu-hai Tu, M. Cristina Marchetti, Hugues Chate, and Francesco Ginelli for many stimulatingly combative discussions; Jean-Francois Joanny for a casual comment that clarified for me the correct way to treat the ordered phase of the flocking model; and the Max-Planck-Institut fur Physik komplexer Systeme, Dresden, and the Centre Emile Borel of the Institut Henri Poincare, Paris, for their hospitality while a portion of this work was being done. In particular I thank Sylvie Lhermitte, Claude Lucet, and Nitdavanh Sriratanakoul, for getting me to the Paris airport despite a transit strike.

[1] C. Reynolds, Computer Graphics 21, 25 (1987); J.L Deneubourg and S. Goss, Ethology, Ecology, Evolution 1, 295 (1989); A. Huth and C. Wissel, in Biological Motion, eds. W. Alt and E. Hoffmann (Springer Verlag, 1990)p. 577-590. B. L. Partridge, Scientific American, 114-123(June 1982). 
[2] W. Loomis, The Development of Dictyostelium discoideum (Academic, New York, 1982); J.T. Bonner, The Cellular Slime Molds (Princeton University Press, Princeton, NJ, 1967).

[3] W.J. Rappel, A. Nicol, A. Sarkissian, H. Levine, W. F. Loomis, Phys. Rev. Lett., 83(6), 1247 (1999).

[4] H. Levine, W. J. Rappel, I. Cohen, Phys. Rev. E, 63, 17101 (2001).

[5] T. Vicsek, Phys. Rev. Lett. 75, 1226 (1995); A. Czirok, H. E. Stanley, and T. Vicsek, J. Phys. A 30, 1375 (1997); T. Vicsek, A. Czirók, E. Ben-Jacob, I. Cohen, and O. Shochet Phys. Rev. Lett. 75, 1226 (1995).

[6] J. Toner and Y. Tu, Phys. Rev. Lett. 75, 4326 (1995).

[7] Y. Tu, M. Ulm and J. Toner, Phys. Rev. Lett. 80, 4819 (1998).

[8] J. Toner and Y. Tu, Phys. Rev. E 58, 4828 (1998).

[9] J. Toner, Y.-h. Tu, and S. Ramaswamy, Ann. Phys. 318, 170 (2005).

[10] N. D. Mermin and H. Wagner, Phys. Rev. Lett. 17, 1133 (1966); P. C. Hohenberg, Phys. Rev. 158, 383 (1967); N. D. Mermin, J. Math. Phys. 8, 1061 (1967).

[11] See, e.g., G. Gregoire, H. Chate, Y. Tu, Phys. Rev. Lett., 86, 556 (2001); G. Gregoire, H. Chate, Y. Tu, Phys. Rev. E, 64, 11902 (2001); G. Gregoire, H. Chate, Y. Tu, Physica D, 181, 157-171 (2003); G. Gregoire, H. Chate, Phys. Rev. Lett., 92(2), (2004).

[12] H. Chate, F. Ginelli, G. Gregoire, and F. Reynaud, Phys. Rev. E 77, 046113 (2008).

[13] S. Ramaswamy and R. A. Simha, Phys. Rev. Lett. 89, 058101 (2002); Phys. Rev. A 306, 262 (2002); R. A. Simha, Ph. D. Thesis, Indian Institute of science, 2003;
Y. Hatwalne, S. Ramaswamy, M. Rao, and R. A. Simha, Phys. Rev. Lett. 92, 118101 (2004).

[14] This should be thought of as an implicit condition on $v_{0}$, since $\alpha$ and $\beta$ can, in general, depend on $|\vec{v}|=v_{0}$.

[15] J. Toner, arXiv:1108.4392 (Condensed Matter), (2011).

[16] See, e.g., E. Bertin, M. Droz, and G. Gregoire, J. Phys. A: Math. Theor. 42, 445001 (2009) arXiv:0907.4688; E. Bertin, M. Droz, and G. Gregoire, Phys. Rev. E 74, 022101 (2006) cond-mat/0601038.

[17] See, e.g., P. Calabrese and P. Parruccini, Nucl.Phys. B 679, 568 (2004).

[18] The speed $\gamma$ may, in some flocks, actually be greater than the mean speed $v_{0}$ of the flock; hence, in some cases, the "slow" bird may have to actually move faster than the other birds. I will describe the different bird in either case as the "slow" bird.

[19] This ignores weak, logarithmic corrections in precisely four dimensions; see [6 9]; in practice, such corrections should be hard to see.

[20] To see this, note that the argument of the scaling function $f_{\Gamma_{L}}$ is $O(1)$ in this regime; hence, $f_{\Gamma_{L}}$ itself should be roughly constant throughout this range of $\vec{q}$.

[21] Of course, this is not exactly true: the slow bird, like the other birds, could make mistakes in following the local direction of flock motion. However, such mistakes would simply add a conventional, "drunkard's walk" lateral wandering (the RMS amplitude of which would scale with time $t$ like $\sqrt{t}$ ) to the much larger wandering due to following the flock that I am computing here. 